\title{
Donor-Sigma-Acceptor Molecules Incorporating a Nonadecyl-Swallowtailed Perylene-Bisimide Acceptor
}

by Lyle D. Wescott and Daniell L. Mattern*

\section{Supporting Information}

$\begin{array}{ll}\text { Procedure for preparation of 10-nonadecanamine } & \text { S2 }\end{array}$

$\begin{array}{ll}\text { IR data for new prepared compounds } & \text { S3 }\end{array}$

$\begin{array}{ll}1 \mathrm{H}-\mathrm{NMR} \text { spectrum for } \mathbf{2 a} & \text { S4 }\end{array}$

13C-NMR spectrum for $\mathbf{2 a} \quad$ S5

HMBC spectrum for 3a (aromatic region) S6

$\begin{array}{ll}\text { HMBC spectrum for } \mathbf{2 3} & \text { S7 }\end{array}$

$\begin{array}{ll}\text { HMBC spectrum for } \mathbf{2 3} \text { (carbonyl region) } & \text { S8 }\end{array}$

HMBC spectrum for 23 (perylene region) $\quad$ S9

$\begin{array}{lr}\mathrm{HMBC} \text { spectrum for } \mathbf{2 3} \text { (phenyl region) } & \text { S10 }\end{array}$

$\begin{array}{ll}\text { HMBC spectrum for } \mathbf{2 3} \text { (methyl region) } & \text { S11 }\end{array}$ 
Procedure for preparation of 10-Nonadecanamine.

10-Nonadecanamine. ${ }^{13}$ A solution of $1.00 \mathrm{~g}(3.54 \mathrm{mmol})$ of 10 -nonadecanone in $10 \mathrm{~mL}$ of ethanol and $5 \mathrm{~mL}$ of pyridine was treated with $0.50 \mathrm{~g}(7.2 \mathrm{mmol})$ of hydroxylamine hydrochloride and then heated on a steam bath for $2 \mathrm{~h}$. The resulting mixture was concentrated by rotary evaporation and the residue was partitioned between 5\% $\mathrm{HCl}(\mathrm{aq})$ and hexanes. The organic layer

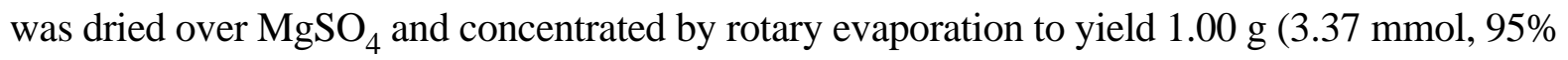
yield) of 10-nonadecanone oxime as an oil.

The oxime was dissolved in $25 \mathrm{~mL}$ of toluene. About $10 \mathrm{~mL}$ was distilled off to dry the system, which was then flushed with $\mathrm{N}_{2}$. Four $\mathrm{mL}$ of $70 \%$ sodium bis(2-methoxyethoxy)aluminum hydride (RedAl) in toluene were added over $5 \mathrm{~min}$. There was immediate gas evolution which subsided as more RedAl was added. The system was refluxed for $2 \mathrm{~h}$. The resulting mixture was carefully added, with stirring, to $20 \mathrm{~mL}$ of $5 \% \mathrm{HCl}$; then $7 \mathrm{~mL}$ of conc $\mathrm{HCl}$ was added to dissolve aluminum salts and render the mixture acidic to litmus. The solution was extracted with hexanes and the organic layer was washed with $5 \% \mathrm{NaOH}$, dried, and concentrated by rotary evaporation to yield $0.88 \mathrm{~g}$ (88\% yield) of 10-nonadecanamine. ${ }^{1} \mathrm{H}-\mathrm{NMR}\left(\mathrm{CDCl}_{3}\right) \delta 0.81(\mathrm{t}, 6 \mathrm{H}), 1.20(\mathrm{~m}, 32 \mathrm{H}), 2.14$ (broad, 2H), 2.68 (m, 1H). IR (KBr): 3372 (w), 3256 (w), 2949 (s), 1463 (s), 1377 (m), 801 (m), $727(\mathrm{~s}) \mathrm{cm}^{-1}$.

13Demmig, S.; Langhals, H. Chem Ber. 1988, 121, 225-230. 
IR data for new prepared compounds.

N,N'-Di-(10-nonadecyl)perylene-3,4,9,10-bis(dicarboximide) (3a). IR (PFE/PE cards): 2920 (m), 2852 (m), 1694 (m), 1650 (s), 1594 (m), 1575 (w), 1455 (w), 1405 (w), 1338 (s), 1252 (m), $1172(\mathrm{w}), 811 \mathrm{~cm}^{-1}$.

N-(10-Nonadecyl)-3,4,9,10-perylenetetracarboxylic acid 3,4-anhydride-9,10-imide (2a). IR (PE card): 1775 (s), 1732 (m), 1703 (m), 1661 (m), 1594 (s), 1406 (m), 1356 (m), 1319 (s), 1251 (m), $1017(\mathrm{~m}), 809(\mathrm{~m}) \mathrm{cm}^{-1}$.

N-(10-Nonadecyl)-N'-(1-pyrenylmethyl)perylene-3,4,9,10-bis(dicarboximide) (4). IR (PE card): 1694 (s), 1658 (s), 1651 (s), 1594 (s), 1436 (w), 1404 (m), 1338 (s), 1260 (s), 1172 (w), 1094 (s), 1021 (s), $846(\mathrm{~m}), 809(\mathrm{~s}) \mathrm{cm}^{-1}$.

N-(10-Nonadecyl)-N'-(4-[1-pyrenyl]butyl)perylene-3,4,9,10-bis(dicarboximide) (5). IR (PE card) 1691 (s), 1658 (s), 1650 (s), 1596 (m), 1463 (w), 1438 (w), 1405 (m), 1339 (s), 1256 (m), $1165(\mathrm{w}), 840(\mathrm{~m}), 808(\mathrm{~m}), 745(\mathrm{~m}) \mathrm{cm}^{-1}$.

N-(10-Nonadecyl)-N'-(2-ferrocenylethyl)perylene-3,4,9,10-bis(dicarboximide) (6). IR (PE card) 1695 (s), 1658 (s), 1650 (s), 1596 (m), 1405 (m), 1343 (m), 1247 (w), 1164 (w), 1127 (w), $1106(\mathrm{w}), 998(\mathrm{w}), 807(\mathrm{~m}), 745(\mathrm{~m}) \mathrm{cm}^{-1}$.

N-(10-Nonadecyl)-N'-(2-phenylethyl)perylene-3,4,9,10-bis(dicarboximide) (7). IR (PE card) 1698 (s), 1651 (s), 1594 (m), 1405 (w), 1342 (s), 1259 (m), 1167 (w), 1074 (w), 1021 (w), $848(\mathrm{~m}), 809$ (s), $745(\mathrm{~m}) \mathrm{cm}^{-1}$.

2-Iodo-N,N,N',N'-tetramethyl-1,4-benzenediamine (16). IR (PE card): 2828 (m), 2778 (m), 1600 (m), 1542 (w), 1501 (s), 1443 (m), 1347 (w), 1306 (m), 1223 (w), 1173 (m), 1140 (w), 1044 (w), 1019 (w), $945(\mathrm{~m}), 837(\mathrm{w}), 803(\mathrm{~m}), 737$ (w), 666 (w) $\mathrm{cm}^{-1}$.

2-(2-Cyanoethenyl)-N,N,N',N'-tetramethyl-1,4-benzenediamine (19). IR (PE card): 2828 (m), 2786 (m), 2363 (w), 2214 (m), 1613 (m), 1559 (w), 1505 (s), 1426 (w), 1356 (w), 1314 (w), 1189 (w), 1169 (w), 1144 (w), 1048 (w), 986 (w), 944 (m), 820 (w) cm-1.

2-(2-Cyanoethyl)-N,N,N',N'-tetramethyl-1,4-benzenediamine (20). IR (PE card): 2836 (s), 2247 (w), 1613 (m), 1513 (s), 1451 (w), 1422 (w), 1356 (w), 1297 (w), 1169 (w), 945 (m), 812 (w) $\mathrm{cm}^{-1}$.

N-(9-Nonadecyl)-N'-(3-[2-(N",N",N"',N"'-tetramethyl-1,4-benzenediaminyl)propyl])perylene-4,5,9,10-bis(dicarboximide) (21). IR (PE card): 1696 (s), 1659 (s), 1596 (s), 1505 (w), $1459(\mathrm{w}), 1405$ (m), 1343 (s), 1256 (w), 1165 (w), 853 (w), 812 (m), 745 (m) cm-1. 
IMIDE ANHYDRIDE, $2 \mathbf{a}$
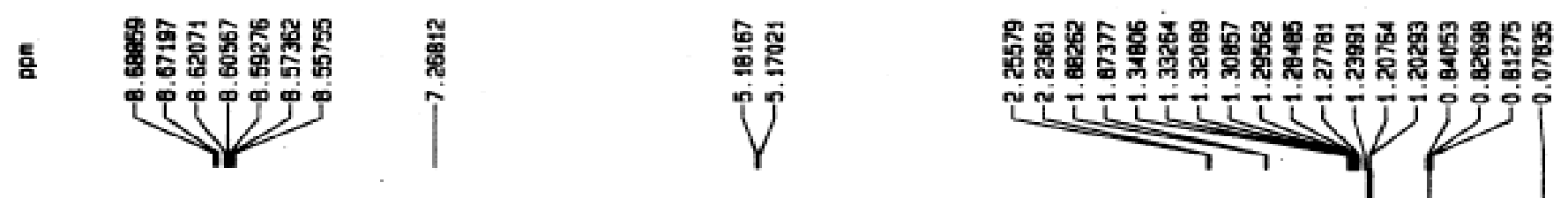

$\sum_{3}^{2}$

(8)

(2) (2)

(28) (6)

pom

9

1.

7

6

5

(1)

' 1 r'r.m.'




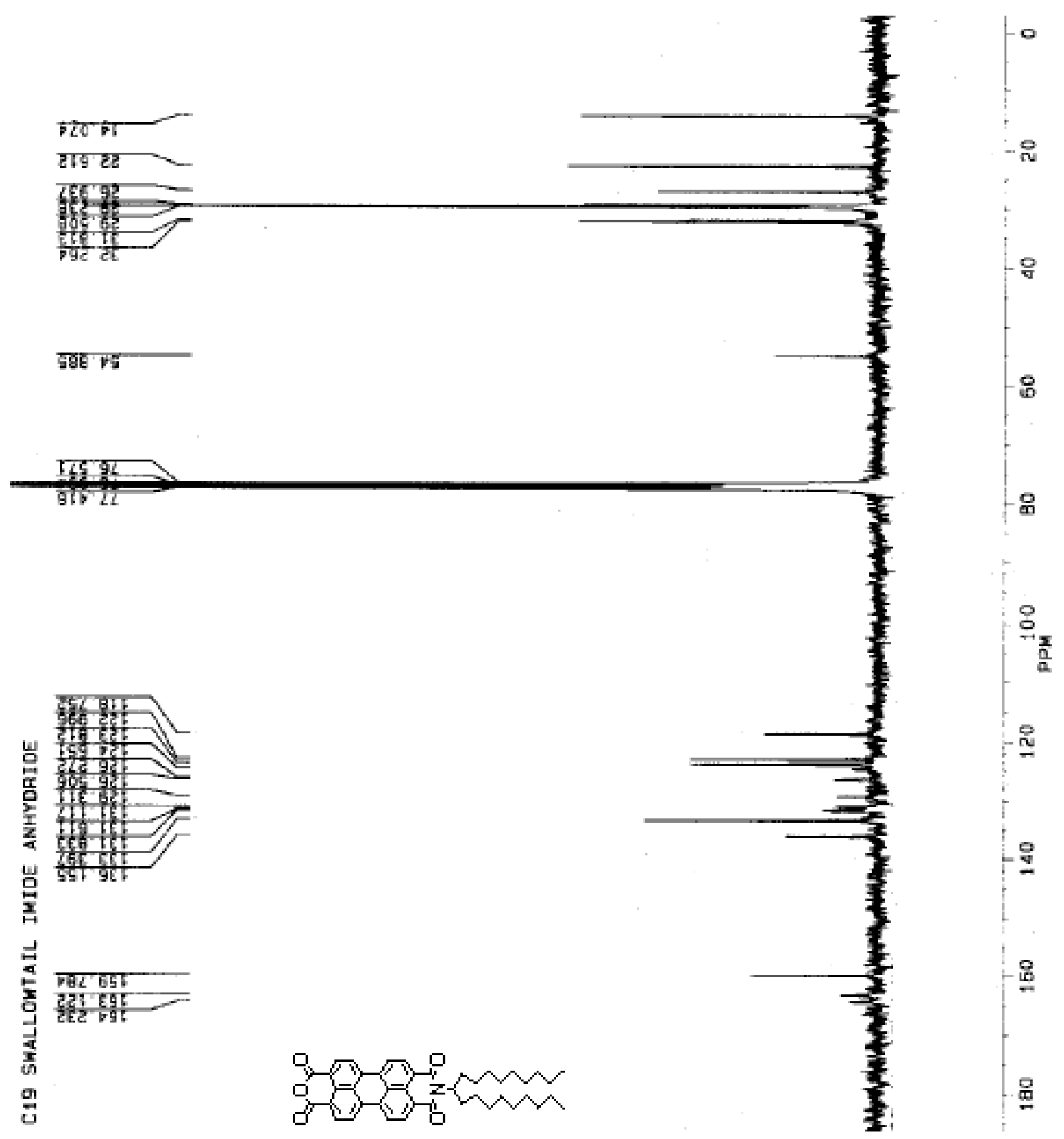


Doubly Swallowtailed Perylenebisimide, 3a

$\mathrm{HMBC}$ spectrum, aromatic region, $60^{\circ} \mathrm{C}$

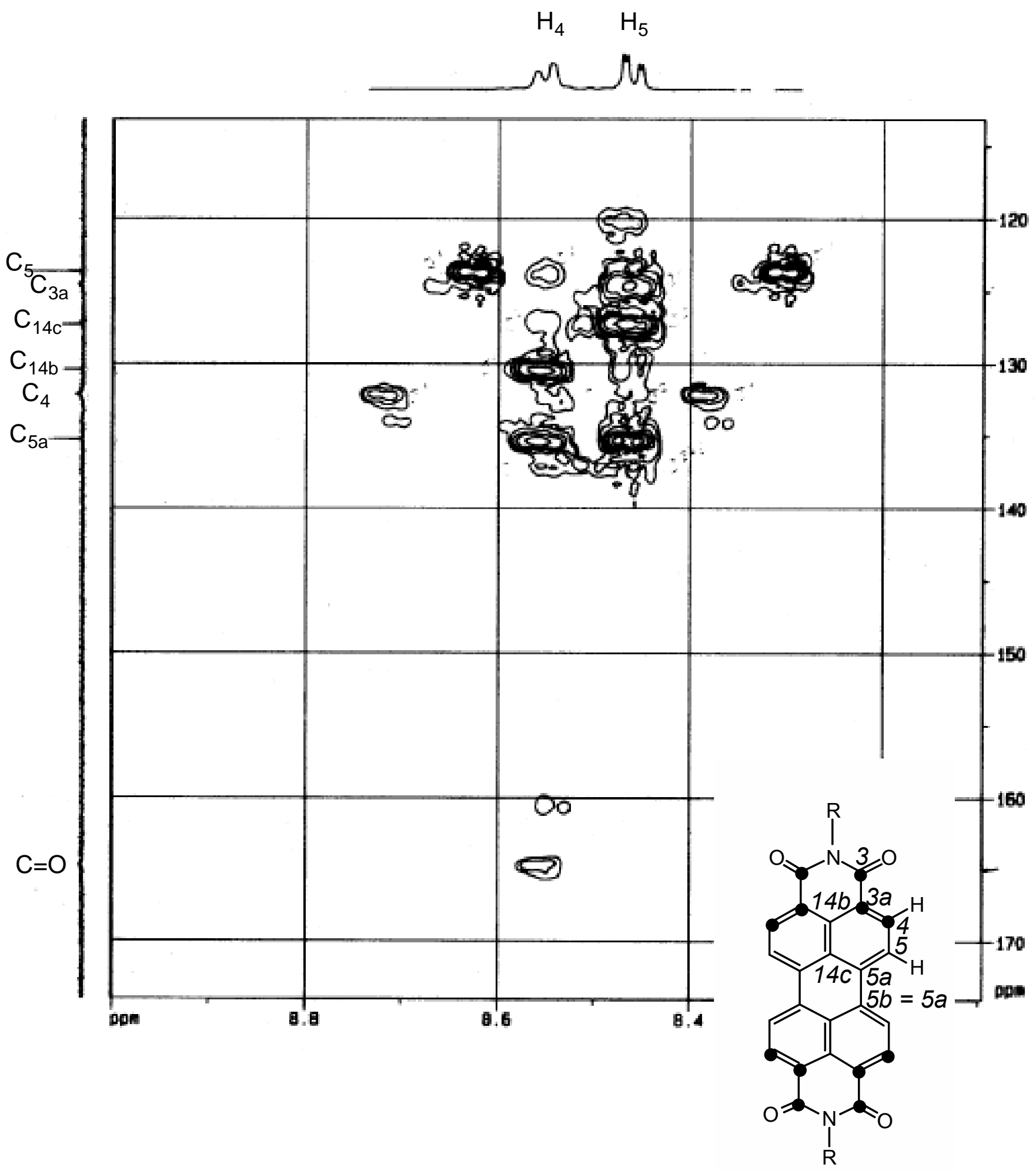


N,N'-Bis-(2,5-di-tert-butylphenyl)perylene-3,4,9,10-bis(dicarboximide), 23

HMBC spectrum

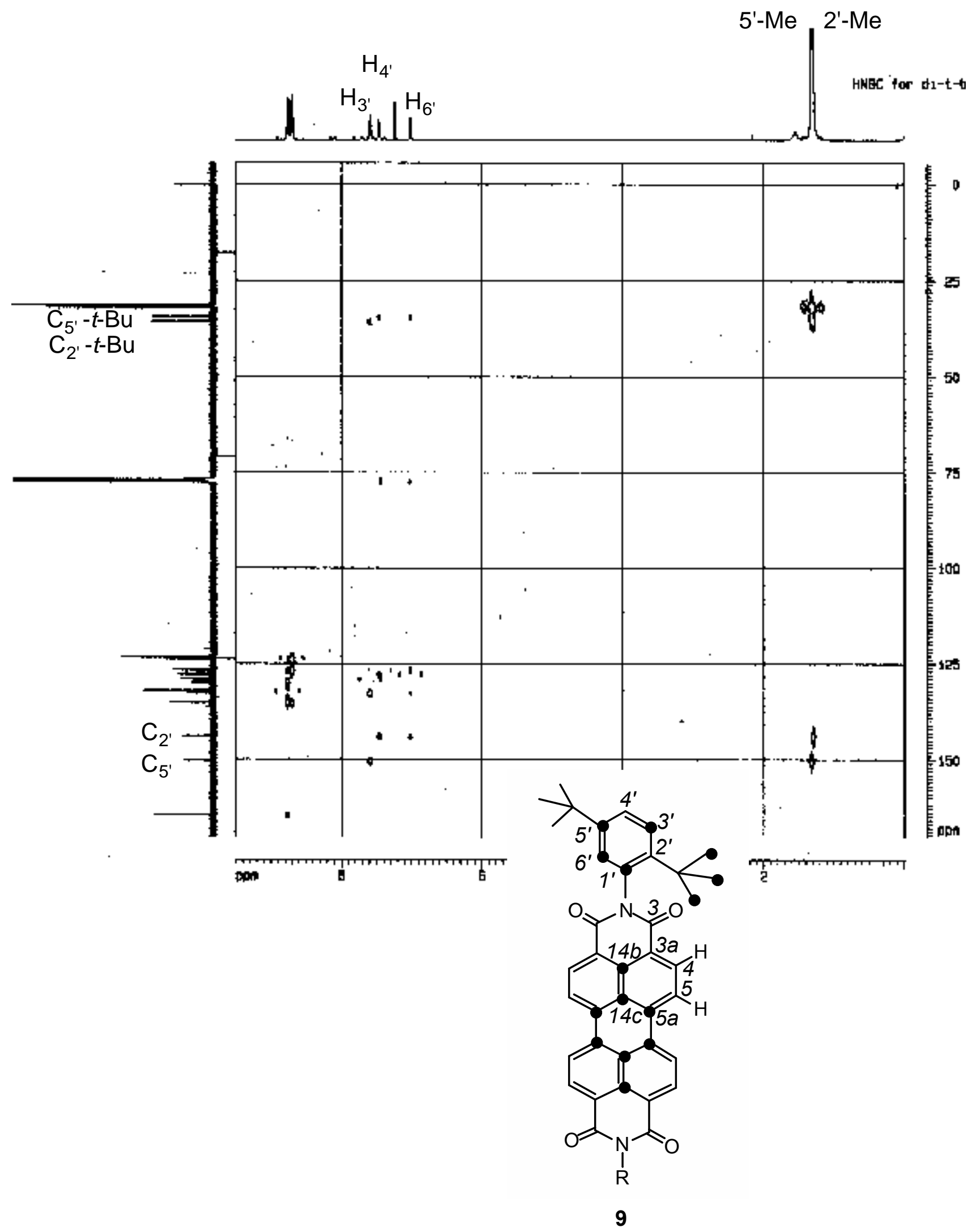


N,N'-Bis-(2,5-di-tert-butylphenyl)perylene-3,4,9,10-bis(dicarboximide), 23

HMBC spectrum, carbonyl

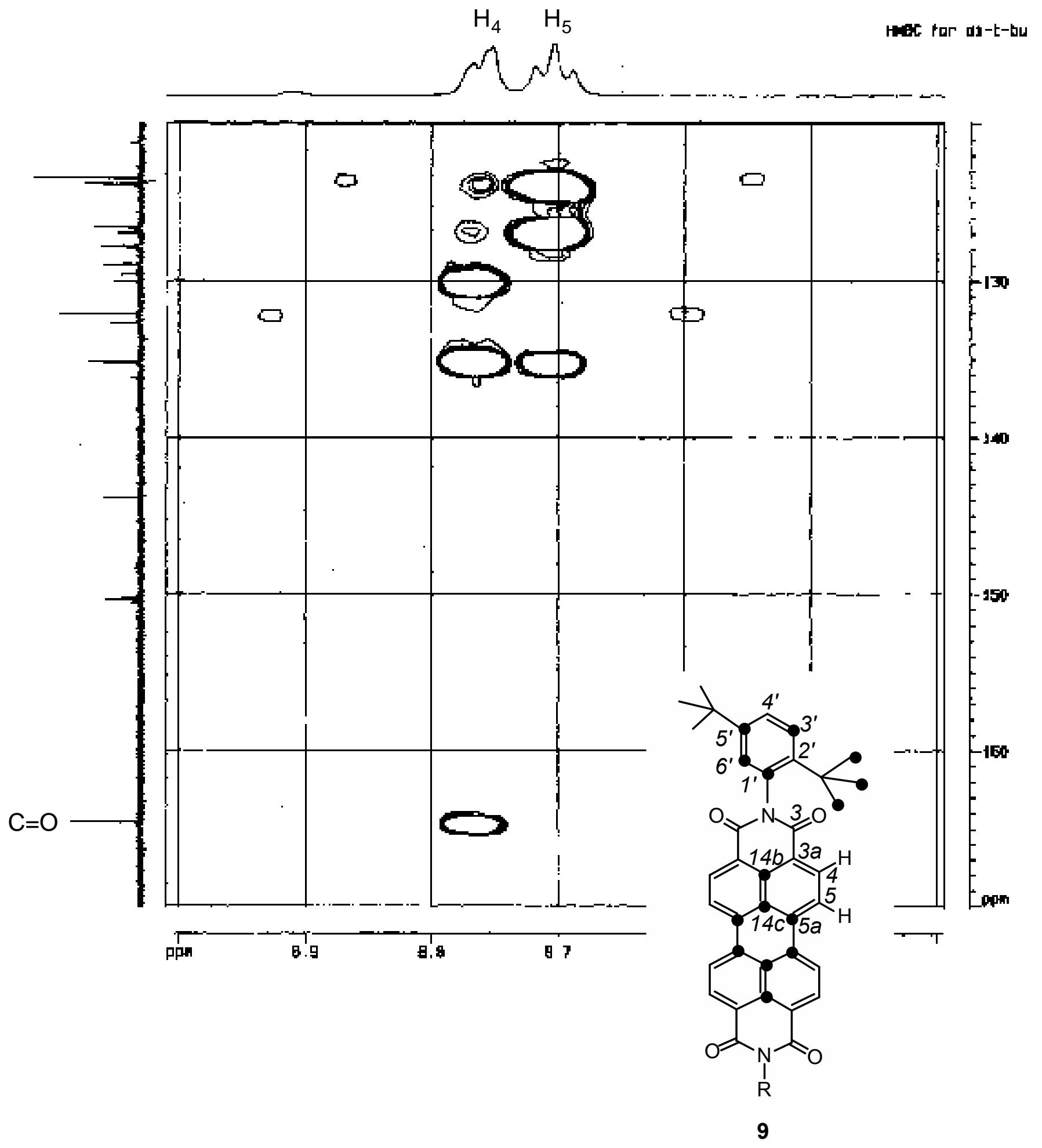


N,N'-Bis-(2,5-di-tert-butylphenyl)perylene-3,4,9,10-bis(dicarboximide), 23 HMBC spectrum, perylene

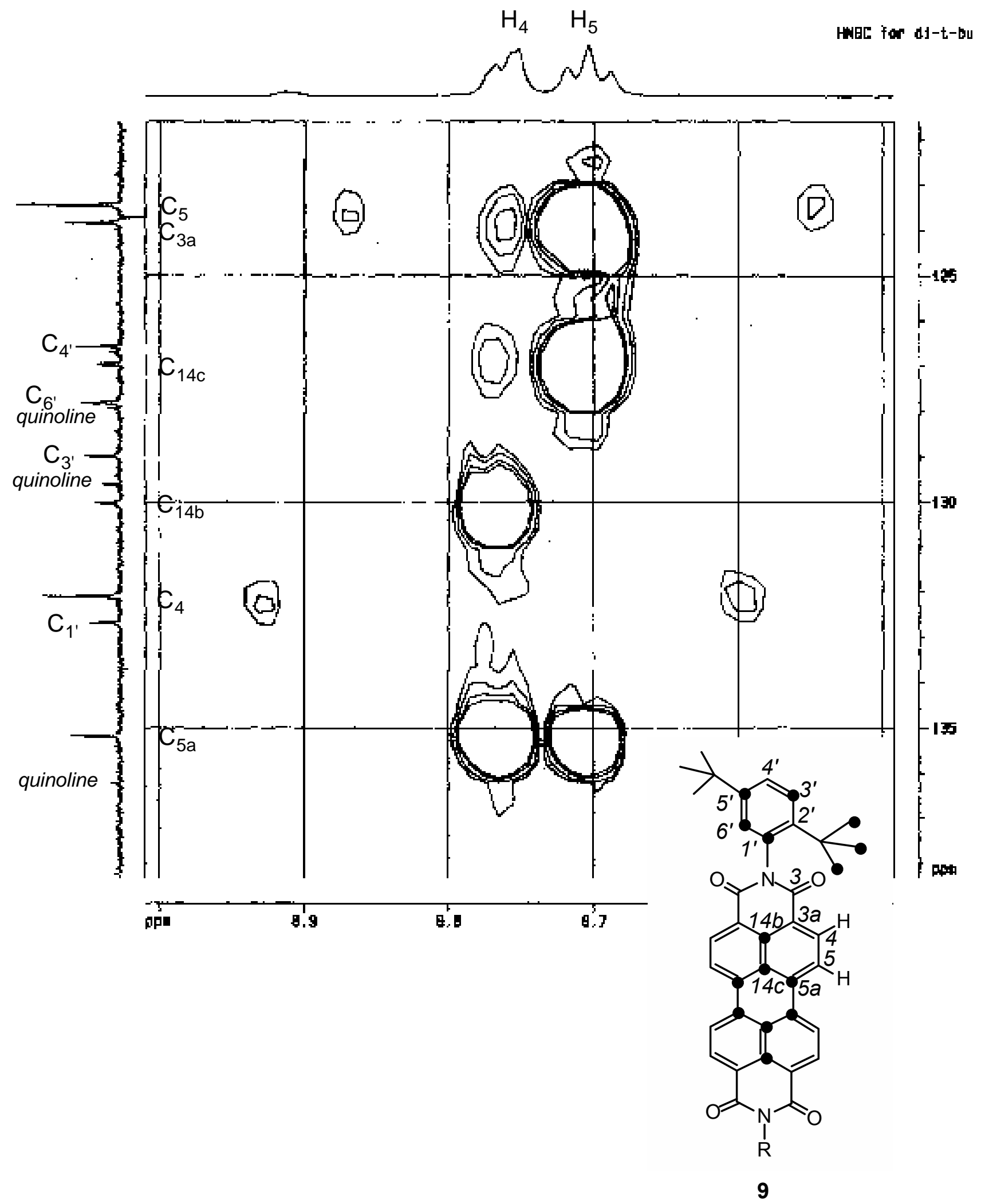


N,N'-Bis-(2,5-di-tert-butylphenyl)perylene-3,4,9,10-bis(dicarboximide), 23 HMBC spectrum, phenyl

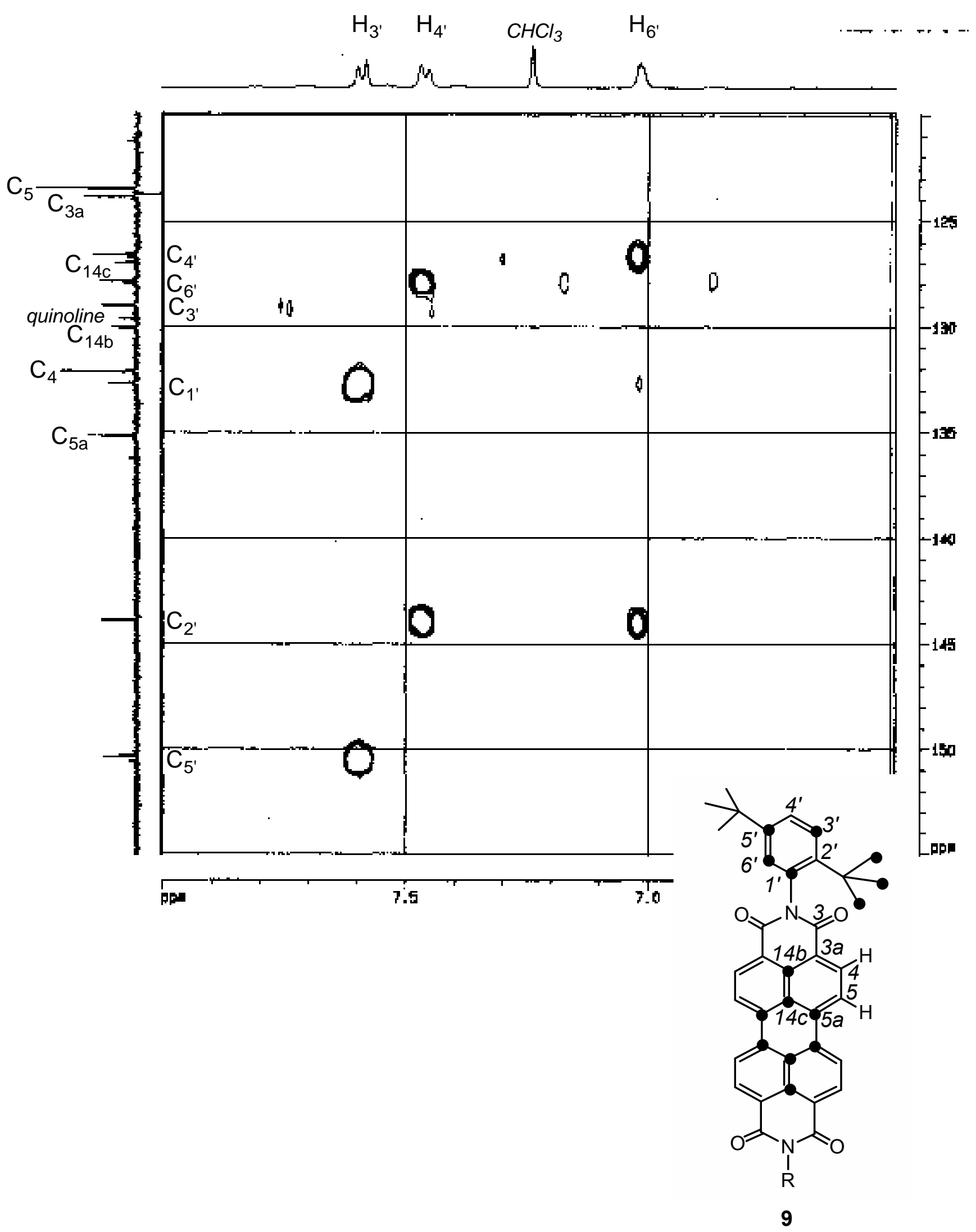


N,N'-Bis-(2,5-di-tert-butylphenyl)perylene-3,4,9,10-bis(dicarboximide), 23

HMBC spectrum, methyl

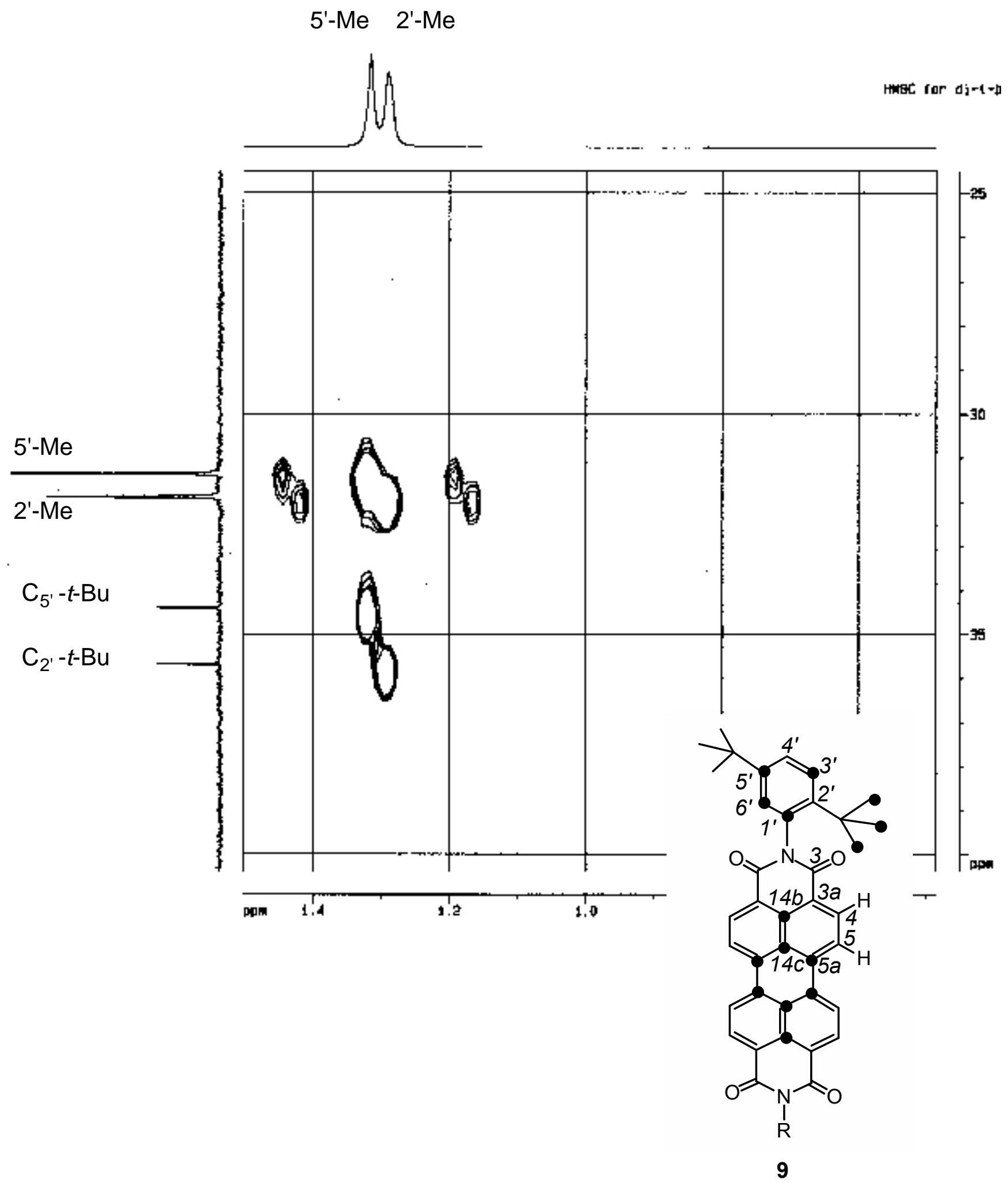

